\title{
TOF-SEMSANS-Time-of-flight spin-echo modulated small-angle neutron scattering
}

\author{
M. Strobl, ${ }^{1}$ A. S. Tremsin, ${ }^{2}$ A. Hilger, ${ }^{3}$ F. Wieder, ${ }^{3}$ N. Kardjilov, ${ }^{3}$ I. Manke, ${ }^{3}$ W. G. Bouwman, ${ }^{4}$ \\ and J. Plomp ${ }^{5}$ \\ ${ }^{1}$ Helmholtz Zentrum Berlin für Materialien und Energie, G-A1, Hahn-Meitner Platz 1, 14109 Berlin, \\ Germany and ESS-AB, Science Division, Stora Algatan 4, 22100 Lund, Sweden \\ ${ }^{2}$ Space Sciences Laboratory, University of California at Berkeley, Berkeley, California 94720, USA \\ ${ }^{3}$ Helmholtz Zentrum Berlin für Materialien und Energie, F-I2, Hahn-Meitner Platz 1, 14109 Berlin, Germany \\ ${ }^{4}$ Faculty of Applied Sciences, Delft University of Technology, Mekelweg 15, NL-2629 JB Delft, \\ The Netherlands \\ ${ }^{5}$ Reactor Institut Delft, Delft University of Technology, Mekelweg 15, NL-2629 JB Delft, The Netherlands
}

(Received 5 December 2011; accepted 24 May 2012; published online 3 July 2012)

\begin{abstract}
We report on measurements of spatial beam modulation of a polarized neutron beam induced by triangular precession regions in time-of-flight mode and the application of this novel technique spin-echo modulated small-angle neutron scattering (SEMSANS) to small-angle neutron scattering in the very small angle range. It is shown that this method can be implemented straight-forwardly in order to extend the accessible size range of structures to be investigated by SANS towards a microscopic scale by applying a divergent beam and measuring the real space correlation function. The novel approach of SEMSANS enables the application of sophisticated sample environment and measurements of magnetic samples (in contrast to the analogue method SESANS). (C) 2012 American Institute of Physics. [http://dx.doi.org/10.1063/1.4730775]
\end{abstract}

\section{INTRODUCTION}

Small-angle neutron scattering (SANS) is one of the most important applications of neutron science. Conventional pinhole collimated SANS instruments are used to investigate bulk structures in a size range of below $1 \mathrm{~nm}$ up to about $10^{2} \mathrm{~nm}$ and are applied to a broad variety of materials from soft matter to hard matter. Limited access to even larger structures, which can be found in many modern materials displaying hierarchical order, biological samples, microporous media, polymers, cements, and colloids to name only a few, could finally only be achieved with extensive focusing devices or elaborated approaches like double-crystal-diffractometer set-ups, which involve further complications and limitations. In addition SANS, in contrast to many other neutron methods could not yet profit significantly from modern pulsed spallation sources, although time-of-flight instruments have been realized. Only the projected long-pulse target station of the European Spallation Source (ESS) might change that situation, due to a time-structure in neutron production, which benefits also low-wavelength resolution techniques like SANS $(\mathrm{d} \lambda / \lambda$ of the order of $10 \%)$. Another method to extend the resolution range of SANS instruments towards the $\mu \mathrm{m}$ range and which has already been realized successfully as a time-offlight (TOF) instrument ${ }^{1}$ is through Larmor encoding of the scattering angle, i.e., spin-echo-SANS (SESANS). ${ }^{2,3}$ The scattering function is in that case measured as a real space correlation function and the detected signal is the final degree of polarization of a divergent neutron beam as a function of what is referred to as the (wavelength dependent) spin-echo length in analogy to the spin-echo time in conventional SE-spectrometers. However, this method requires keeping control of the neutron spin throughout the instrument, i.e., not only in the spin-echo regions before and after the sample but also in the sample region. This in turn hinders application of sophisticated sample environment as well as it prohibits or at least significantly complicates measurements of magnetic sample materials. A novel approach first proposed by Gähler ${ }^{4}$ can overcome these drawbacks by allowing for limiting all spin manipulations to the area upstream of the sample position. As has been shown accordingly by Bouwman et al., the utilization of inclined magnetic field regions-using the geometry of half the number of magnets of a SESANS instrument-and tuned magnetic fields (Fig. 1), a transversal spatial intensity modulation can be induced to the neutron beam through spin analyses. Here, it will be demonstrate how this technique can be realized in a TOF mode and that it can be applied for very small angle neutron scattering efficiently.

\section{THEORY}

For the full derivation of the description for SANS measurements we refer to Ref. 6, but we give a short review of the essential equations to describe the method. A set-up of two triangular precession regions $B_{1}$ and $B_{2}$ with the same geometry but opposite field direction at distances $\mathrm{L}_{1}$ and $\mathrm{L}_{2}$ from the detector, respectively, and their fields adjusted according to $\mathrm{L}_{1} \mathrm{~B}_{1}=\mathrm{L}_{2} \mathrm{~B}_{2}$ induce a modulation of the polarization of an originally homogenously polarized neutron beam at the detector position, which depends only on the transversal position on the detector, but not on the origin of the neutron. Consequently, a divergent beam can be used and in case an analyzer is placed at any position along $\mathrm{L}_{2}$ a corresponding sinusoidal spatial beam modulation can be detected with a position sensitive detector with sufficient spatial resolution. The wavelength dependent period of the modulation can be calculated to be

$$
\zeta=\pi \tan \theta_{0} /\left(\mathrm{c} \lambda\left(\mathrm{B}_{2}-\mathrm{B}_{1}\right)\right)
$$



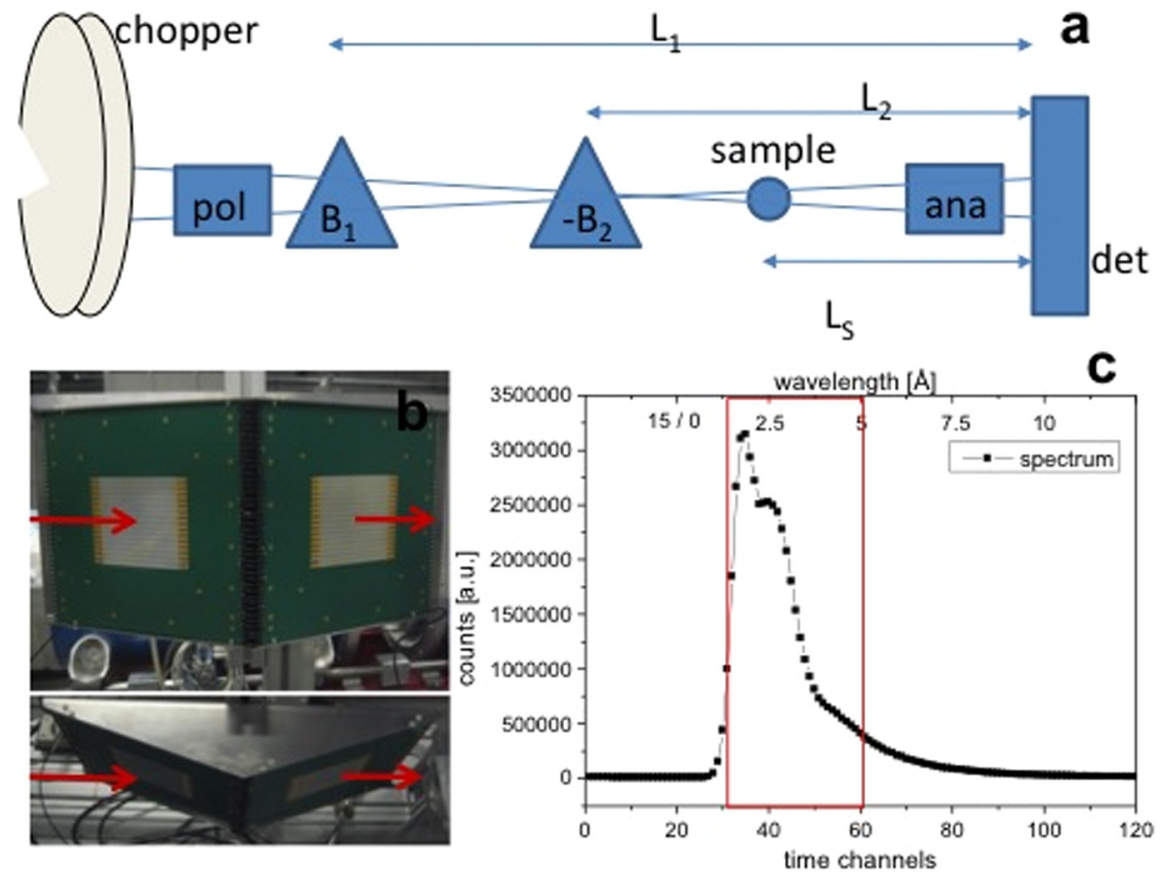

FIG. 1. A schematic sketch of the instrument layout including the double chopper, a polarizer (pol), the triangular magnetic field regions $\left(B_{1}\right.$ and $\mathrm{B}_{2}$ ), sample position, polarization analyzer (ana), and the 2D position sensitive and time resolving detector (det); (a) two photographs of the triangular field set up constructed of current sheets (b) and the measured spectrum provided by the beamline highlighting the utilized part (c).

where $\theta_{0}$ is the inclination angle between the normal of the interface of the field region and the neutron beam axis. The dependence of the period on the wavelength implies the requirement of a detector with sufficient spatial and time resolution in order to implement the technique in a TOF mode. The spin-echo length, i.e., the correlation length probed with a certain set of parameters, can be defined in analogy to SESANS but becomes position dependent (along the beam) and when the sample is placed somewhere along $L_{2}$ (no matter if before or behind the analyzer) with a distance $\mathrm{L}_{\mathrm{S}}$ to the detector it can be written to be

$$
\delta^{\mathrm{SE}}=\mathrm{c} \lambda^{2} \mathrm{~L}_{\mathrm{s}}\left(\mathrm{B}_{2}-\mathrm{B}_{1}\right) / \pi \tan \theta_{0}=\lambda \mathrm{L}_{\mathrm{s}} / \zeta
$$

and displays a wavelength square dependence. However, in contrast to SESANS not polarization but the measured modulation amplitude A constitutes the measurement signal as

$$
\mathrm{A}\left(\delta^{\mathrm{SE}}\right) / \mathrm{A}_{0}=\mathrm{e}^{\sigma \mathrm{t}\left(\mathrm{G}\left(\delta_{\mathrm{SE}}\right)-1\right)} .
$$

Here, $\sigma$ and $\mathrm{t}$ are the scattering cross section and the sample thickness, respectively, while

$$
G\left(\delta^{S E}\right)=\frac{1}{\sigma k_{0}^{2}} \int_{\text {det }} d Q_{y} d Q_{x} \frac{d \sigma(\vec{Q})}{d \Omega} \cos \left(Q_{y} \delta^{S E}\right)
$$

is the SESANS correlation function with $\mathrm{k}_{0}$ being the incident wavevector and $\mathrm{Q}$ is the scattering vector. ${ }^{5,6}$

\section{MEASUREMENTS AND RESULTS}

A corresponding spin-echo modulated small-angle neutron scattering (SEMSANS) setup has been set up on the WESP beamline at the $2 \mathrm{MW}$ research reactor of RID of TU Delft. The beamline offers an optical blind double chopper system, ${ }^{7}$ which, when the choppers are set to a distance of $\mathrm{z}_{0}=0.4 \mathrm{~m}$, offers for a chopper to detector distance of
$\mathrm{L} \approx 6.2 \mathrm{~m}$ a constant wavelength resolution of $\mathrm{d} \lambda / \lambda=6.5 \%$ for a spectrum that peaks at $2.1 \AA$ (Fig. 1). The pulse frequency was $40 \mathrm{~Hz}$. The triangular precession regions were built up by two current sheets connected under an angle of $140^{\circ}\left(\theta_{0} \approx 20^{\circ}\right)$ each and were installed in the guide field available at the WESP beamline. The precession regions are similar to the coils developed by Pynn and co-workers. ${ }^{8}$ The current sheets have been supplied with $8.5 \mathrm{~A}$ and $18 \mathrm{~A}$ (scan 1) current corresponding to magnetic fields of $\mathrm{B}_{1} \approx 2.3 \mathrm{mT}$ and $\mathrm{B}_{2} \approx 4.52 \mathrm{mT}$, respectively, and with $3.7 \mathrm{~A}$ and $8.7 \mathrm{~A}$ (scan 2), i.e., $1.15 \mathrm{mT}$ and $2.26 \mathrm{mT}$, and the corresponding distances to the detector were $\mathrm{L}_{1}=2.635 \mathrm{~m}$ and $\mathrm{L}_{2}=1.365 \mathrm{~m}$. The beam was polarized and analyzed by means of multi channel supermirror devices. For detection a micro-channel plate (MCP) based detector with fast Medipix readout electronics was used in order to achieve sufficient time and spatial resolution. ${ }^{9,10} 120$ images corresponding to time slices of $200 \mu$ s have been recorded for every chopper pulse. Sufficient intensities were recorded in optimum case for $8 \mathrm{~h}$ measurement times only for 30 time channels of the resulting spatial resolved histogram data corresponding to wavelengths between $1.39 \AA$ and $5.28 \AA$ with an increment of $0.13 \AA$. Measurements have been performed for both field settings with and without sample. As a reference sample, a quartz glass cuvette filled with monodisperse polystyrene (PS-DVB) spheres with diameters of $136 \mathrm{~nm}$ dispersed in $\mathrm{D}_{2} \mathrm{O}$ with a concentration of $12.4 \mathrm{wt}$. $\%$ and a sample thickness of $4 \mathrm{~mm}$ was used. The modulation observed in vertical direction is due to the channels of the polarization analyzer which was, owed to constructive restrictions at the beamline, placed directly in front of the imaging detector. Spin-echo beam modulation was induced according to the geometry of the field regions in horizontal direction and hence the image data could be integrated vertically during data reduction (Fig. 2). The resulting sinusoidal modulation functions, as shown in Fig. 3, for every wavelength slice and measurement have been fitted 

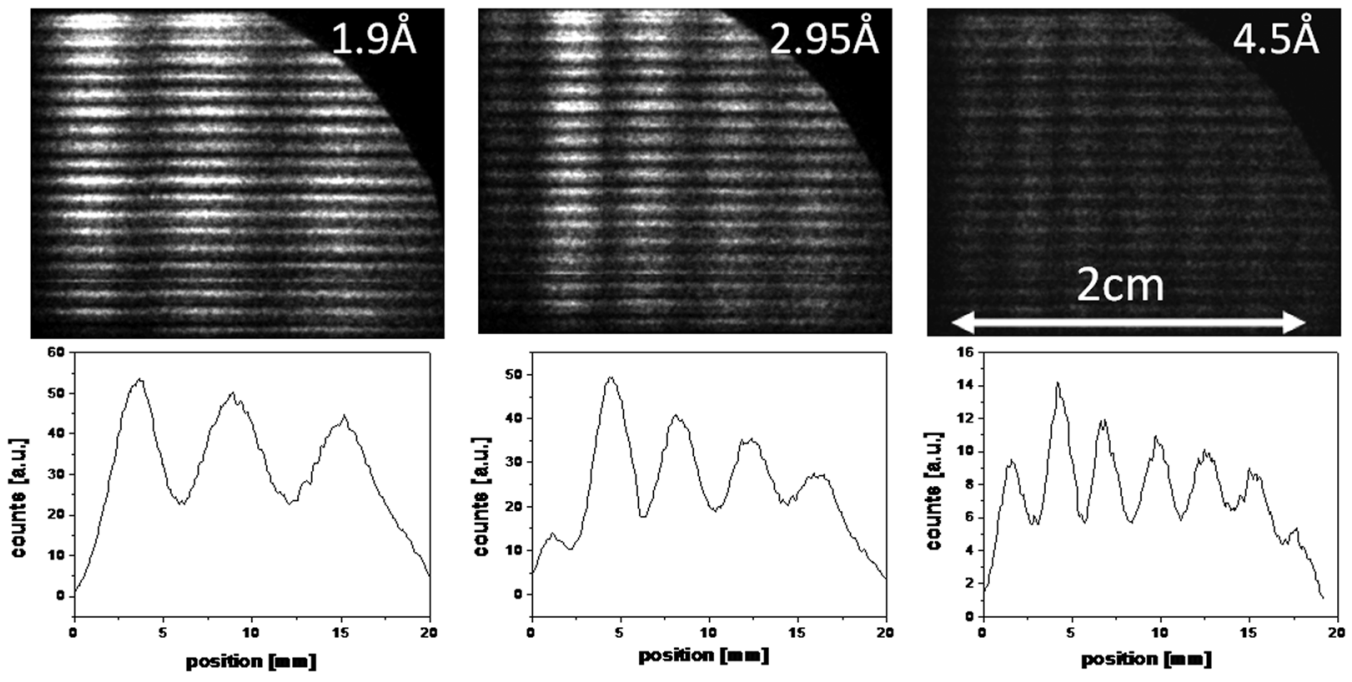

FIG. 2. Recorded images of the modulated beam for specific wavelengths and the corresponding line profiles (integrated over the image height; note that the horizontal stripes are due to the structure of the polarization analyzer, without any meaning for the measurements).

and provided corresponding values of the modulation frequencies and amplitudes from which together with the known sample distance $\mathrm{L}_{\mathrm{S}} \approx 1 \mathrm{~m}$ the resulting SE-lengths $\delta^{\mathrm{SE}}(\lambda)$ and relative modulation amplitudes $\mathrm{A}\left(\delta^{\mathrm{SE}}, \lambda\right) / \mathrm{A}_{0}(\lambda)$, respectively, could be extracted (Fig. 3) after an attenuation correction has been performed. The SESANS correlation function is retrieved according to Eq. (3) by taking the logarithm and by dividing the normalized amplitude by the thickness and the wavelength squared, since the scattering cross section is proportional to this. The two scans performed on the sample have been merged and re-binned, which results in one final SEM-
SANS curve of the sample. A size range of one order of magnitude has been probed between about $16 \mathrm{~nm}$ and $160 \mathrm{~nm}$. However, it can be seen easily from Eqs. (1) and (2) that, e.g., an extension of the wavelength band, an increase of magnetic field or using multiple triangular field regions, enables an extension towards the $\mu \mathrm{m}$ range. A precondition is the availability of a detector with corresponding spatial resolution, but the used MCP detector has proven to achieve resolutions down to approximately $14 \mu \mathrm{m}^{9,10}$

The correlation function data are in good agreement with theoretical predictions ${ }^{11}$ for the sample. Only a few
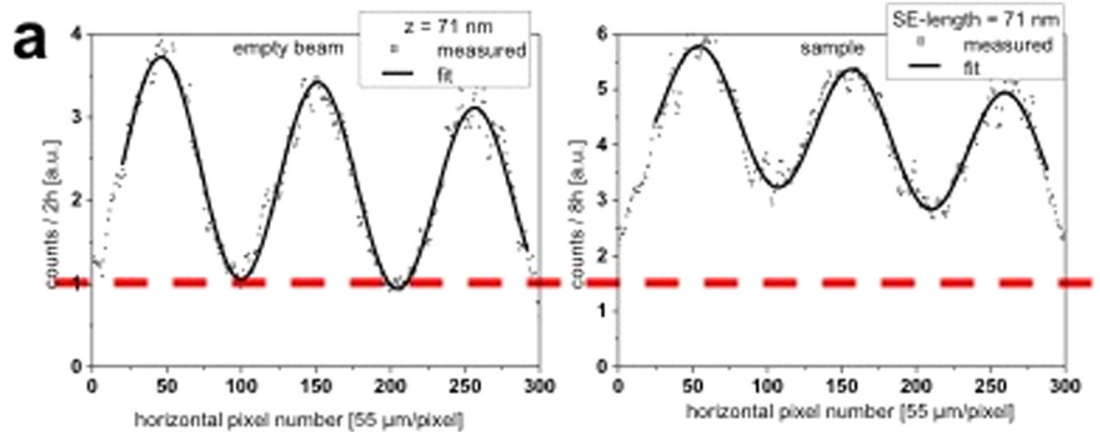

b

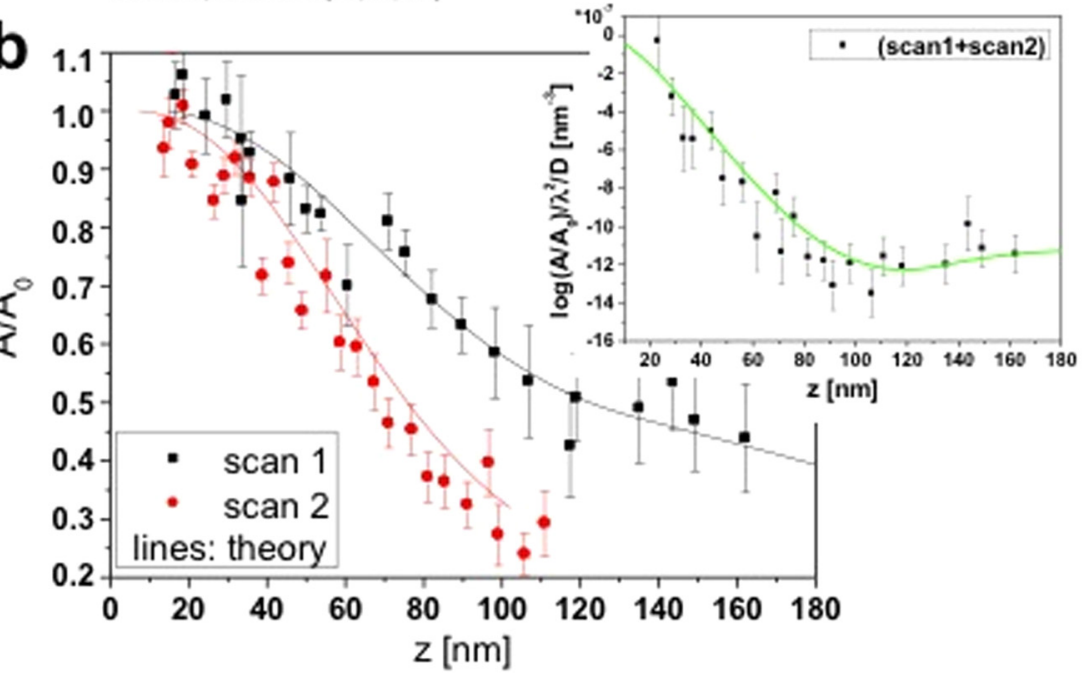

FIG. 3. These graphs show the raw data and their analysis to obtain the projected correlation function. (a) Examples of corresponding detected beam modulations without (left) and with sample (right) scaled from zero to maximum and hence directly representing the damping of the modulation amplitude (note that the dashed line marking the minima in the empty beam measurement). (b) From these modulations, the normalised amplitudes are retrieved versus spinecho length. The line is the calculated amplitude. The inset in (b) shows the SESANS correlation function $\mathrm{G}$ calculated by taking the logarithm and normalising with wavelength and sample thickness. 
measurement points display a clear deviation, these, however, are such where the sine fit did not provide good correlation. At spin-echo length values close to the size of the investigated PS spheres the slope of the correlation function displays a significant change corresponding to the characteristic saturation or minimum at that value in a spin-echo scattering curve. ${ }^{11,12}$ Then, the curve displays the upturn which indicates the detection of the nearest neighbor particle-particle correlation.

\section{CONCLUSION}

In summary, we have shown (1) how already a simple field set-up allows for inducing spatial beam modulation, (2) that a realization in TOF mode can be established straightforwardly, and (3) that the corresponding SEMSANS method is well suited for scattering investigations in the very small angle range. From these results achieved at a not specifically optimized TOF beamline at a low flux reactor source and with a thermal neutron spectrum the authors conclude that TOFSEMSANS promises an outstanding potential for efficient investigations of structures in size ranges overlapping and significantly extending that of conventional SANS instruments, overcoming the flux limitations in that range, and that realizations of dedicated instrumentation are promising as standalone instruments and due to simplicity also as optional add-on devices at SANS instruments. In comparison to SESANS, the instrumental effort and spatial requirements are clearly reduced and the possibility to place the sample downstream of all required spin manipulating components allows in the case of SEMSANS for sophisticated sample environment as well as for investigating magnetic systems. In addition, in analogy to corresponding SESANS approaches the application of SEMSANS can be extended to specular and off-specular neutron reflectometry as well as for grazing incidence SANS (GISANS). ${ }^{13}$ Furthermore, the lack of substantial collimation requirements enables to investigate large area samples with spatial resolution, i.e., in a dark-field imaging mode. ${ }^{14,15}$

\section{ACKNOWLEDGMENTS}

The authors are thankful to Dr. Yan Lu for preparing and providing sample material. This work has been funded by the BMBF Project No. 05E10CB1 (HZB partition) and by the NMI3 program.

${ }^{1}$ J. Plomp, V. O. de Haan, R. M. Dalgliesh, S. Langridge, and A. A. van Well, Thin Solid Films 515, 5732 (2007).

${ }^{2}$ W. G. Bouwman, W. Stam, T. V. Krouglov, J. Plomp, S. V. Grigoriev, W. H. Kraan, and M. Th. Rekveldt, Nucl. Instrum. Methods Phys. Res. A 529, 16 (2004).

${ }^{3}$ J. Plomp, V. O. de Haan, R. M. Dalgliesh, S. Langridge, and A. A. van Well, Physica B 397, 76 (2007).

${ }^{4} \mathrm{R}$. Gähler, A Certain Class of Beam Modulation Techniques and Its Potential Applications (PNCMI Polarized Neutron School, 2006).

${ }^{5}$ W. G. Bouwman, Ch. P. Duif, and R. Gähler, Phys. B 404, 2585 (2009).

${ }^{6}$ W. G. Bouwman, Ch. P. Duif, J. Plomp, A. Wiedenmann, and R. Gähler, Physica B 406, 2357 (2011).

${ }^{7}$ A. A van Well, Phys. B 180-181, 959 (1992).

${ }^{8}$ R. Pynn, W. T. Lee, P. Stonaha, V. R. Shah, A. L. Washington, B. J. Kirby, C. F. Majkrzak, and B. B. Maranville, Rev. Sci. Instrum. 79, 063901 (2008).

${ }^{9}$ A. S. Tremsin, J. V. Vallerga, J. B. McPhate, O. H. W. Siegmund, W. B. Feller, L. Crow, and R. G. Cooper, Nucl. Instrum. Methods Phys. Res. A $\mathbf{5 9 2 ,} 374$ (2008).

${ }^{10}$ A. S. Tremsin, J. B. McPhate, J. V. Vallerga, O. H. W. Siegmund, W. B. Feller, E. Lehmann, and M. Dawson, Nucl. Instrum. Methods Phys. Res. A 652, 400 (2011).

${ }^{11}$ T. Krouglov, I. M. de Schepper, W. G. Bouwman, and M. Th. Rekveldt, J. Appl. Crystallogr. 36, 117 (2003).

${ }^{12}$ R. Andersson, L. van Heijkamp, I. M. de Schepper, and W. G. Bouwman, J. Appl. Crystallogr. 41, 868 (2008).

${ }^{13}$ M. Th. Rekveldt, J. Appl. Crystallogr. 36, 1301 (2003).

${ }^{14}$ M. Strobl, C. Grünzweig, A. Hilger, I. Manke, N. Kardjilov, C. David, and F. Pfeiffer, Phys. Rev. Lett. 101, 123902 (2008).

${ }^{15}$ M. Strobl, N. Kardjilov, A. Hilger, I. Manke, and J. Banhart, J. Phys. D 42, 243001 (2009). 